This item was submitted to Loughborough's Research Repository by the author.

Items in Figshare are protected by copyright, with all rights reserved, unless otherwise indicated.

\title{
Game theoretic analysis for MIMO radars with multiple targets
}

PLEASE CITE THE PUBLISHED VERSION

http://dx.doi.org/10.1109/TAES.2016.150699

PUBLISHER

IEEE

VERSION

VoR (Version of Record)

\section{PUBLISHER STATEMENT}

This work is made available according to the conditions of the Creative Commons Attribution 3.0 Unported (CC BY 3.0) licence. Full details of this licence are available at: http://creativecommons.org/licenses/by/3.0/

\section{LICENCE}

CC BY 3.0

\section{REPOSITORY RECORD}

Deligiannis, Anastasios, Sangarapillai Lambotharan, and Jonathon Chambers. 2016. "Game Theoretic Analysis for MIMO Radars with Multiple Targets". figshare. https://hdl.handle.net/2134/21897. 


\section{Game Theoretic Analysis for MIMO Radars With Multiple}

\section{Targets}

ANASTASIOS DELIGIANNIS, Student Member, IEEE SANGARAPILLAI LAMBOTHARAN, Senior Member, IEEE Loughborough University, Loughborough, UK

JONATHON A. CHAMBERS, Fellow, IEEE

Newcastle University, Newcastle upon Tyne, UK

This paper considers a distributed beamforming and resource allocation technique for a radar system in the presence of multiple targets. The primary objective of each radar is to minimize its transmission power while attaining an optimal beamforming strategy and satisfying a certain detection criterion for each of the targets. Therefore, we use convex optimization methods together with noncooperative and partially cooperative game theoretic approaches. Initially, we consider a strategic noncooperative game (SNG), where there is no communication between the various radars of the system. Hence each radar selfishly determines its optimal beamforming and power allocation. Subsequently, we assume a more coordinated game theoretic approach incorporating a pricing mechanism. Introducing a price in the utility function of each radar/player enforces beamformers to minimize the interference induced to other radars and to increase the social fairness of the system. Furthermore, we formulate a Stackelberg game by adding a surveillance radar to the system model, which will play the role of the leader, and hence the remaining radars will be the followers. The leader applies a pricing policy of interference charged to the followers aiming at maximizing his profit while keeping the incoming interference under a certain threshold. We also present a proof of the existence and uniqueness of the Nash equilibrium (NE) in both the partially cooperative and

Manuscript received September 28, 2015; revised February 8, 2016; released for publication July 8, 2016.

DOI. No. 10.1109/TAES.2016.150699.

Refereeing of this contribution was handled by F. Gini.

This work was supported by the Engineering and Physical Sciences Research Council (EPSRC) Grant EP/K014307/1 and the MOD University Defence Research Collaboration (UDRC) in Signal Processing.

Authors' addresses: A. Deligiannis, S. Lambotharan, Loughborough University, Wolfson School of Mechanical, Manufacturing and Electrical Engineering, Epinal Way, Loughborough, LE11 3TU UK; J. Chambers, School of Electrical and Electronic Engineering, Newcastle University, Newcastle upon Tyne NE1 7RU, UK. Corresponding author is A. Deligiannis, E-mail: (a.deligiannis@lboro.ac.uk)

This work is licensed under a Creative Commons Attribution 3.0 License. For more information, see http://creativecommons.org/licenses/by/3.0.

0018-9251/16/\$26.00 C 2016 IEEE noncooperative games. Finally, the simulation results confirm the convergence of the algorithm in all three cases.

\section{INTRODUCTION}

Multiple-input multiple-output (MIMO) radar is an innovative technology that has raised expectations over the last decade that it will provide substantial improvements to the currently used radar systems. The main characteristic that allows MIMO radar to offer superior capabilities as compared with other radar regimes is its waveform diversity, which implies that MIMO radar can use multiple antennas to simultaneously transmit several orthogonal waveforms and multiple antennas to receive the reflected signals from the targets [1]. There are two principal MIMO radar schemes considered in the literature, the systems incorporating colocated antennas and those that consist of widely separated antennas (bistatic, multistatic) [2,3]. The leading fields of research within MIMO radar technology are beamformer and waveform design, detection optimization, and radar imaging [4-6]. Succeeding the advances in those fields, the main advantages offered by MIMO radar are higher angular resolution, direct applicability of adaptive array techniques, multiple target detection, and the ability to obtain spatial diversity in the target's radar cross section (RCS). Nevertheless, one substantial drawback in a multiple target, distributed radar system, that has not yet been completely resolved, is the multiple source interference imposed at the receivers of each radar. More specifically, the interradar, ${ }^{1}$ the intraradar, ${ }^{2}$ and the clutter interference lead to reduced efficiency and performance degradation of the radar system. Hence, an optimal beamforming and power allocation strategy is crucial as it minimizes the interference in between the radars of the same organization, while preserving a detection criterion. Game theory is a natural and effective tool for modeling this kind of interactions, as it offers a mathematical framework of conflict and cooperation between intelligent, self-interested, and rational players.

The increasing need for independent, autonomous, and decentralized communication systems has sparked much interest in using game theoretic techniques in the communication literature [7]. More specifically, the aforementioned distributed, multistatic beamforming and resource allocation problem in radar systems can be compared with similar issues raised in multicell wireless systems in communication applications [8-16]. In [8], the authors introduced the idea of joint beamforming and power control, proposing an iterative algorithm to simultaneously obtain the optimal beamforming and power vectors. The incorporation of game theory in this context then rapidly became a focal point in

\footnotetext{
${ }^{1}$ Cross channel (direct) and indirect interference induced among different radars.

${ }^{2}$ Interference imposed from the transmitters to the receivers of the same radar when detecting two or more different targets.
} 
communications research [9-14]. The majority of this literature considers the technique of strategic noncooperative games (SNG), where each player selfishly maximizes its payoff function, given the strategies of the other players. The authors of [9] exploited an iterative water-filling algorithm to reach the Nash equilibrium (NE) in a noncooperative, distributed, multiuser power control problem. Since each player greedily optimizes its utility function, the equilibrium might not be the Pareto-optimal solution. Introducing pricing policies to the system resources leads to a more Pareto-efficient solution and increases the social welfare of the system. A pricing regime that is a linear function of the transmit power was studied in [10]. Another example of pricing the transmit power of each player is considered in [11], whereas in [12] and [13] the pricing policy is applied on the intercell interference among the players. In [14], the authors consider the optimization of a set of precoding matrices at each node of a multichannel, multiuser cognitive radio MIMO network in order to minimize the total transmit power of the network, while applying a pricing scheme based on global information. Cooperative game theoretic techniques combined with a two-level Stackelberg game were utilized in [15] to address the problem of relay selection and power allocation without the knowledge of channel state information (CSI). Finally, the authors in [16] formulated a Stackelberg Bayesian game to obtain the optimal power allocation for a two-tier network, while applying an interference constraint at the leader and considering channel gain uncertainty.

Game theory is also an efficient tool to overcome various problems that arise in radar systems. In particular, the authors in [17] approached the problem of polarimetric waveform design by considering a zero-sum game between an opponent and the radar system engineer. The zero-sum game was also used in [18] to investigate the interaction between a MIMO radar and an intelligent target, that applies jamming techniques. Potential game theory was exploited in [19] with the main objectives of optimal waveform design and maximization of the signal-to-interference plus noise ratio (SINR). A noncooperative game theoretic per antenna power optimization based on signal-to-disturbance ratio (SDR) estimation with a desired SINR constraint was investigated in [20]. Noncooperative game theory was also employed in [21] to facilitate the power control problem in a radar network. To address the power allocation problem the authors of [22] used a cooperative game approach and exploited the Shapley value solution scheme.

In this paper, inspired by the aforementioned game theoretic methods applied in communications [10-14], although reinvestigated to adapt to the radar case, we have developed a broad game theoretic analysis for the optimal beamforming and resource allocation problem in a MIMO tracking radar system with multiple targets. Initially, we consider an SNG, where each radar/player greedily optimizes the beamforming and power allocation vectors in two stages. In the first stage, the optimal transmit and receive beampatterns are designed by exploiting convex optimization techniques in a power minimization problem, while attaining a certain detection criterion. After designing the optimal beampatterns, the primary joint beamforming and resource allocation problem reduces to a power only minimization game. Thus, in the second stage of the game we obtain the best response strategy of a radar in an SNG setup and show that it is a standard function [32], which proves the uniqueness of the NE, similar to the work in [12] for wireless communication applications.

The fact that each radar acts selfishly and does not take into account the damage it may inflict to other radars, through interradar interference, leads to a solution that may not be optimal from a social welfare point of view. Since we assume that the radars belong to the same organization, it is safe to consider some sort of cooperation and introduce a pricing policy to all players in order to minimize the interference induced to other radars. More specifically, the radars are encouraged to steer their beams in directions that cause less damage to other players, which results in a more Pareto-optimal solution.

In order to complete our radar model, we incorporate a surveillance radar as part of the previously studied MIMO tracking radar system. The main application of the surveillance radar is to continuously search the operating area for new incoming targets. By adding a surveillance radar, our hybrid radar system is capable of both acquiring new targets and tracking every target in an operating field. However, all radars operate simultaneously and hence the tracking radars interfere with the surveillance radar and increase the probability of false alarm. In order to secure the smooth operation of the system, we set a maximum limit of interference induced at the surveillance radar. In order to achieve both the target SINR and to guarantee the interference limit at the surveillance radar, we utilize a Stackelberg game approach. In particular, the surveillance radar is the leader and the MIMO tracking radars are the followers in the hierarchy of the game. We next introduce the system model.

\section{SYSTEM MODEL}

We consider a multistatic radar network that consists of $K$ separate radars each consisting of $M$ transmit/receive antennas. The set of radars is denoted by $C=\{1, \ldots, K\}$. In order to complete the model, $L$ targets are assumed in the far-field of the radars, so that the main objective for each radar is to attain a specific detection performance for every target using the minimum possible transmission power. In the noncooperative design of the multistatic radar network, the radars try to minimize their transmission power independently, having full knowledge of the uplink and the downlink channels of their own radar, whereas they have no knowledge of the interradar channel gains. Since we consider that the radars belong to the same organization, the design of the model is not competitive, as there is no deliberate interference between the radars. However, as we do not assume communication between 


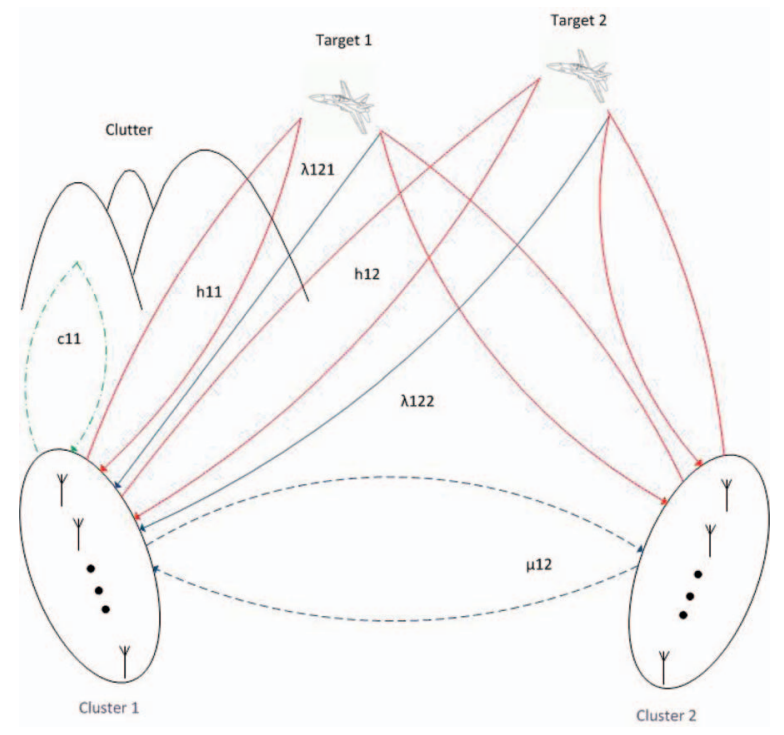

Fig. 1. Multistatic MIMO radar network with two radars and two targets.

radars a noncooperative game is appropriate. An example of a multistatic radar network with two radars, two targets, and clutter in the far-field is illustrated at Fig. 1.

In order to detect the $l^{t h}$ target, the transmit array of the $k^{\text {th }}$ radar emits the $l^{\text {th }}$ element of the independent, predesigned waveform vector $\boldsymbol{\psi}_{k}(t)=\left[\boldsymbol{\psi}_{k 1}(t), \ldots, \boldsymbol{\psi}_{k L}(t)\right]^{T}$ of size $L \times 1$, which satisfies the orthogonality condition $\int_{T_{0}} \boldsymbol{\psi}_{k}(t) \boldsymbol{\psi}_{k}^{H}(t) d t=\mathbf{I}_{L}$, where $(\cdot)^{T}$ denotes the transpose operator, $t$ refers to the time index within the radar pulse, $T_{0}$ is the radar pulsewidth, $\mathbf{I}_{L}$ is the $L \times L$ identity matrix, and $(\cdot)^{H}$ denotes the Hermitian transpose operator. Thus, the waveforms corresponding to different targets are not correlated, i.e., $\int_{T_{0}} \boldsymbol{\psi}_{k l}(t) \boldsymbol{\psi}_{k l^{\prime}}(t) d t=0$, where $l \neq l^{\prime}$. We assume that the waveform vector maintains the orthogonality condition for a set of acceptable time delays $\tau_{a}, \tau_{a}{ }^{\prime}$ and Doppler frequency shifts $f_{D a}, f_{D a^{\prime}}$, such as [23]

$$
\begin{aligned}
& \int_{T_{0}} \boldsymbol{\psi}_{k a}\left(t-\tau_{a}\right) \boldsymbol{\psi}_{k a^{\prime}}\left(t-\tau_{a^{\prime}}\right) e^{j 2 \pi\left(f_{D a}-f_{D a^{\prime}}\right) t} d t \\
& \quad \approx \begin{cases}1, & \text { if } a=a^{\prime} \\
0, & \text { if } a \neq a^{\prime}\end{cases}
\end{aligned}
$$

However, if the waveforms arrive with considerable delays and Doppler shifts, we may expect nonzero correlation between waveforms. This correlation factor is denoted as

$$
\varrho_{k, l, l^{\prime}}\left(\tau_{l, l^{\prime}}\right)=\int_{T_{0}} \boldsymbol{\psi}_{k l}(t) \boldsymbol{\psi}_{k l^{\prime}}\left(t+\tau_{l, l^{\prime}}\right) e^{j 2 \pi(\Delta f) t} d t
$$

where $\tau_{l, l^{\prime}}$ is the relevant delay of the waveform returned from the $l^{\text {th }}$ target as compared with the delay of the waveform returned from the $l^{\prime t h}$ target. The relative difference in Doppler frequency is given by $\Delta f=f_{D l}-f_{D l^{\prime}}$. This introduces interference between the signals returning from different targets, as discussed later.
The $M \times 1$ vector which consists of the complex elements of the signal transmitted from the $k^{\text {th }}$ radar and intended for the $l^{\text {th }}$ target is of the form

$$
\mathbf{x}_{k l}(t)=\mathbf{w}_{t(k, l)} \boldsymbol{\psi}_{k l}(t)
$$

where $\mathbf{w}_{t(k, l)}$ is the $M \times 1$ transmit beamforming vector from radar- $k$ to target- $l$. Hence, the overall transmitted signal from radar- $k$ is

$$
\mathbf{x}_{k}(t)=\sum_{l=1}^{L} \mathbf{x}_{k l}(t)=\sum_{l=1}^{L} \mathbf{w}_{t(k, l)} \boldsymbol{\psi}_{k l}(t)
$$

As depicted in Fig. 1, $\mathbf{h}_{k l}$ is the channel gain vector from target- $l$ to radar- $k, \mathbf{c}_{k l}$ denotes the interfering signal returns from the clutter when the $k^{\text {th }}$ radar tags target $l$. The cross-channel gain between radar- $k$ and radar- $i$ is denoted as $\boldsymbol{\mu}_{k i}$ and $\boldsymbol{\lambda}_{k i j}$ represents the interradar interfering signal channel at the $k^{\text {th }}$ radar echoing from the $j^{\text {th }}$ target and emitted from the $i^{\text {th }}$ radar. The uplink and downlink parts of the path gains can be obtained by the following equations with respect to the transmit beamforming vectors and the receive beamforming vectors, respectively:

$$
\begin{gathered}
\mathbf{h}_{t(k l)}=\mathbf{b}\left(\theta_{k l}\right) \mathbf{w}_{t(k, l)}^{H} \mathbf{a}\left(\theta_{k l}\right) \beta_{l} \\
\mathbf{h}_{r(k l)}=\mathbf{b}\left(\theta_{k l}\right) \mathbf{w}_{r(k, l)}^{H} \mathbf{a}\left(\theta_{k l}\right) \beta_{l} \\
\mathbf{c}_{t(k l)}=\mathbf{b}\left(\theta_{c l(k)}\right) \mathbf{w}_{t(k, l)}^{H} \mathbf{a}\left(\theta_{c l(k)}\right) \beta_{c l} \\
\mathbf{c}_{r(k l)}=\mathbf{b}\left(\theta_{c l(k)}\right) \mathbf{w}_{r(k, l)}^{H} \mathbf{a}\left(\theta_{c l(k)}\right) \beta_{c l} \\
\boldsymbol{\mu}_{t(k i)}=\sum_{j=1}^{L} \mathbf{b}\left(\theta_{r a d(k, i)}\right) \mathbf{w}_{t(k, j)}^{H} \mathbf{a}\left(\theta_{r a d(i, k)}\right) \\
\boldsymbol{\mu}_{r(k i)}=\sum_{j=1}^{L} \mathbf{b}\left(\theta_{\operatorname{rad}(k, i)}\right) \mathbf{w}_{r(k, j)}^{H} \mathbf{a}\left(\theta_{\operatorname{rad}(i, k)}\right) \\
\lambda_{t(k i j)}=\mathbf{b}\left(\theta_{k i}\right) \mathbf{w}_{t(i, j)}^{H} \mathbf{a}\left(\theta_{i j}\right) \beta_{j} \\
\lambda_{r(k i j)}=\mathbf{b}\left(\theta_{k i}\right) \mathbf{w}_{r(i, j)}^{H} \mathbf{a}\left(\theta_{i j}\right) \beta_{j}
\end{gathered}
$$

where $\mathbf{w}_{r(k, l)}$ is the $M \times 1$ receive weight vector for radar- $k$ when aimed at target $l, \beta_{l}$ is the complex amplitude proportional to the RCS of target $l, \beta_{c l}$ denotes the RCS amplitude of the clutter, and $\mathbf{a}\left(\theta_{k l}\right)$ and $\mathbf{b}\left(\theta_{k l}\right)$ are the $M \times 1$ transmit and receive steering vectors for radar- $k$, respectively, as defined below:

$$
\begin{aligned}
\mathbf{a}\left(\theta_{k l}\right) & =\left[1, e^{j \frac{2 \pi}{\lambda} d \sin \left(\theta_{k l}\right)}, \ldots, e^{j \frac{2 \pi}{\lambda}(M-1) d \sin \left(\theta_{k l}\right)}\right]^{T} \\
\mathbf{b}\left(\theta_{k l}\right) & =\left[1, e^{j \frac{2 \pi}{\lambda} d \sin \left(\theta_{k l}\right)}, \ldots, e^{j \frac{2 \pi}{\lambda}(M-1) d \sin \left(\theta_{k l}\right)}\right]^{T}
\end{aligned}
$$

where $d$ is the distance between the adjacent antennas and is considered the same for all radars, $\theta_{k l}$ is the azimuth direction of target $l$ by considering radar- $k$ as reference, $\theta_{c l(k)}$ is the direction of the clutter as seen from the $k^{\text {th }}$ radar and $\theta_{\operatorname{rad}(k, i)}$ is the direction of radar- $i$ as observed 
from radar- $k$, and $\lambda$ is the wavelength of the transmitted signal. From the definition, it is apparent that the transmit and receive steering vectors are equal, as the uplink and downlink channels remain constant over the duration of a full game.

By matched-filtering at the receiver of radar- $k$ each of the orthogonal waveforms $\psi_{k l}\left(t-\tau_{l}\right) e^{j 2 \pi f_{D l} t}, l=1, \ldots, L$, the desired received signal for the detection of target- $l$ is obtained by

$$
y_{\text {des }(k l)}=\mathbf{w}_{r(k, l)}^{H} \mathbf{h}_{t(k l)}
$$

Considering a distributed, multistatic, and multitarget radar scheme, the detection of a target is deteriorated by direct and collateral interradar interference, in addition to the interference induced by the signals intended for other targets by the same radar, the clutter effect, and the noise power. As a result, the interference signal can be modeled as

$$
\begin{aligned}
y_{\text {inter } f(k l)}= & \left(\sum_{j \neq l}^{L} \mathbf{w}_{r(k, l)}^{H} \mathbf{h}_{t(k j)} \varrho_{k, l, j}\left(\tau_{l, j}\right)\right. \\
& +\sum_{m \neq k}^{K} \sum_{j=1}^{L} \mathbf{w}_{r(k, l)}^{H} \lambda_{t(k m j)} \varrho_{k, l, m, j}\left(\tau_{l, j}\right) \\
& \left.+\sum_{m \neq k}^{K} \mathbf{w}_{r(k, l)}^{H} \boldsymbol{\mu}_{t(k m)}+\sum_{i=1}^{L} \mathbf{w}_{r(k, l)}^{H} \mathbf{c}_{t(k i)}+\hat{n}\right)
\end{aligned}
$$

where $\varrho_{k, l, m, j}\left(\tau_{l, j}\right)$ denotes the correlation factor between the waveform emitted from the $k^{\text {th }}$ radar and echoed by the $l^{\text {th }}$ target and the waveform emitted from the $m^{\text {th }}$ radar but echoed by the $j^{\text {th }}$ target.

Since we defined the desired and interfering signals for radar- $k$ regarding target- $l$ in (1) and (2), the relevant SINR is straightforwardly defined as

$$
\operatorname{SINR}_{k l}=\frac{\left\|y_{\text {des }(k l)}\right\|^{2}}{\left\|y_{\text {inter } f(k l)}\right\|^{2}}
$$

where $\|\cdot\|$ denotes the Euclidian norm.

Using the above system model, the next section describes the game theoretic formulation of the proposed scheme.

\section{BEAMFORMER DESIGN AND POWER ALLOCATION GAME}

\section{A. Game Theoretic Formulation}

In order to determine the optimal transmit/receive beamformers and power allocation between the radars, we incorporate an SNG. The various radars are considered as players, and therefore the player set is denoted by $C=\{1$, $\ldots, K\}$. Consider the transmit beamforming weight vector matrix $\mathbf{W}_{t(k)}=\left\{\mathbf{w}_{t(k, 1)}, \ldots, \mathbf{w}_{t(k, L)}\right\}$ as the strategy of player- $k$ and the matrix $\mathbf{W}_{t(-k)}$ as the strategy chosen by the other players. Hence, we define the acceptable strategy set for radar- $k$ as

$$
\mathcal{P}_{k}\left(\mathbf{W}_{t(-k)}\right)=\left\{\mathbf{W}_{t(k)} \in \mathbb{C}^{M \times L} \mid \operatorname{SINR}_{k l} \geq \gamma_{k l}, \forall l\right\}
$$

where $\gamma_{k l}$ is the desired SINR for target- $l$ when targeted from the antennas of radar- $k$. The decision on the desired SINR depends on the probabilities of misdetection $P_{m d}$ and false alarm $P_{f a}$, which are derived from the following equations [24, 25]:

$$
\begin{gathered}
P_{m d}\left(\xi_{k l}\right)=\left(1-\xi_{k l}\right)^{N-1} \\
P_{f a}\left(\mathrm{SINR}_{k l}, \xi_{k l}\right)=1-\left(1-\frac{\xi_{k l}}{1-\xi_{k l}} \frac{1}{1+N \operatorname{SINR}_{k l}}\right)^{1-N}
\end{gathered}
$$

where $\xi_{k l}$ denotes the threshold of the generalized likelihood ratio test (GLRT), applied to determine if there is absence or presence of a target [25] and $N$ is the number of samples used for the GLRT. We define a specific design parameter $\varepsilon_{k l}$ to set an upper bound on the tolerance regarding $P_{m d}$ and $P_{f a}$. Hence, the optimum $\operatorname{SINR}_{k l}$ for each radar regarding each target can be determined as $[20,21]$

$$
\begin{aligned}
& \gamma_{k l}^{*}=\min \left\{\operatorname{SINR}_{k l} \mid \exists \xi_{k l} \in[0,1]\right. \\
& \text { s.t. } \left.P_{m d}\left(\xi_{k l}\right)+P_{f a}\left(\operatorname{SINR}_{k l}, \xi_{k l}\right) \leq \varepsilon_{k l}\right\} .
\end{aligned}
$$

It is evident from (3) that the $\operatorname{SINR}_{k l}$ for player- $k$ is a function of the beamforming weight vectors (which include transmission power) of all players. Hence, the set of admissible strategies $\mathcal{P}_{k}\left(\mathbf{W}_{t(-k)}\right)$ for radar- $k$ depends on the beamforming weight matrix $\mathbf{W}_{t(-k)}$ of every other player (radar).

The last component required to complete the game is the utility function for each player, which is defined as $u_{k}\left(\mathbf{W}_{t(k)}\right)=\left\|\mathbf{W}_{t(k)}\right\|_{F}^{2}$ representing the transmit power of player- $k$, where $\|\cdot\|_{F}$ denotes the Frobenius norm. The game is summarized as

$$
\mathcal{G}=\left\langle C,\left\{\mathcal{P}_{k}\left(\mathbf{W}_{t(-k)}\right)\right\}_{k \in C},\left\{u_{k}\left(\mathbf{W}_{t(k)}\right)\right\}_{k \in C}\right\rangle
$$

In the SNG considered, given the beamforming strategies of the other players, each player selfishly minimizes its power allocation subject to a predefined detection criterion. As a result, the best response strategy for player- $k$ is the result of the following optimization:

$$
\begin{aligned}
& \min _{\mathbf{W}_{t(k)}}\left\|\mathbf{W}_{t(k)}\right\|_{F}^{2} \\
& \text { s.t. } \frac{\left|\mathbf{w}_{t(k, l)}^{H} \mathbf{h}_{r(k l)}\right|^{2}}{\sum_{j \neq l}^{L}\left|\mathbf{w}_{t(k, l)}^{H} \mathbf{h}_{r(k j)}\right|^{2}+\sum_{i=1}^{L}\left|\mathbf{w}_{t(k, l)}^{H} \mathbf{c}_{r(k i)}\right|^{2}+r_{-k l}} \geq \gamma_{k l}, \forall l
\end{aligned}
$$

where $\mathbf{r}_{-k}=\left[r_{-k 1}, \ldots, r_{-k L}\right]^{T}$ is the total interference induced by all other radars except radar- $k$ plus the additive white Gaussian noise (AWGN) from the environment vector. For target- $l$, it is defined as $r_{-k l}=$ $\sum_{m \neq k}^{K} \sum_{j=1}^{L}\left|\mathbf{w}_{t(k, l)}^{H} \lambda_{r(k m j)}\right|^{2}+\sum_{m \neq k}^{K}\left|\mathbf{w}_{t(k, l)}^{H} \boldsymbol{\mu}_{r(k m)}\right|^{2}+\sigma_{n}^{2}$.

One of the main objectives of this work is to investigate whether the game $\mathcal{G}$ converges to a stable point, where no player can profit by unilaterally changing its beamforming strategy, as it will lead to higher power consumption to achieve the same SINR for every target. Such a point is an NE and for the game considered, it is 
defined as the strategy set $\left\{\mathbf{W}_{t(1)}^{*}, \ldots, \mathbf{W}_{t(K)}^{*}\right\}$ where

$$
u_{k}\left(\mathbf{W}_{t(k)}^{*}\right) \leq u_{k}\left(\mathbf{W}_{t(k)}\right), \quad \forall \mathbf{W}_{t(k)} \in \mathcal{P}_{k}\left(\mathbf{W}_{t(-k)}^{*}\right), \forall k \in C
$$

In the next section we determine the optimal beampatterns and investigate the best response strategy. We also prove the existence and uniqueness of the NE of the game $\mathcal{G}$.

\section{B. Convex Optimization Beamforming and the Best Response Strategy}

Convex optimization has been widely utilized in the radar beamforming literature. Most of the work concentrates on designing the beamforming vectors in order to approximate a desired beampattern, decided by the target position [26-29]. In the first stage of this analysis, we determine the optimal beampattern for every radar corresponding to each of the targets using convex optimization techniques. After securing the optimal beampatterns, each player should just allocate the minimum possible transmission power, while minimizing the interradar interference and achieving a certain detection performance.

The optimal transmit beampatterns for each radar can be designed by solving the following optimization problem:

$$
\begin{aligned}
& \min _{\mathbf{W}_{t(k)}} \sum_{l=1}^{L}\left\|\mathbf{w}_{t(k, l)}\right\|^{2} \\
& \text { s.t. } \frac{\left|\mathbf{w}_{t(k, l)}^{H} \mathbf{h}_{r(k l)}\right|^{2}}{\sum_{j \neq l}^{L}\left|\mathbf{w}_{t(k, l)}^{H} \mathbf{h}_{r(k j)}\right|^{2}+\sum_{i=1}^{L}\left|\mathbf{w}_{t(k, l)}^{H} \mathbf{c}_{r(k i)}\right|^{2}+r_{-k l}} \geq \gamma_{k l}, \forall l
\end{aligned}
$$

The optimization in (5) can be converted to semidefinite programming (SDP) using the rank relaxation method and solved as in [30] and [31]. The optimal receive weight vectors can be found using generalized eigenvector techniques.

CLAIM 1 The optimal transmit and receive beampatterns are independent of the interradar interference $\mathbf{r}_{-k}$.

PROOF The proof can be found in Appendix A.

Hence, when the radars reallocate the power of transmission, the interradar interference plus noise vector $\mathbf{r}_{-k}$ is modified. From claim 1, radar- $k$ retains the optimal beampatterns derived from (5), however reallocates only its transmission power for each target, in order to achieve the detection criterion. This observation is similar to that considered in wireless communication applications [12], regardless of the appearance of additional clutter in the denominator of the SINR equation in (5). As a result, after obtaining the optimal transmit/receive beamforming vectors, we can reformulate the initial optimization problem (4) as the following power minimization problem:

$$
\begin{aligned}
& \min _{p_{k 1}, \ldots, p_{k L}} \sum_{l=1}^{L} p_{k l} \\
& \text { s.t. } \frac{p_{k l}\left|\hat{\mathbf{w}}_{t(k, l)}^{H} \mathbf{h}_{r(k l)}\right|^{2}}{\sum_{j \neq l}^{L} p_{k j}\left|\hat{\mathbf{w}}_{t(k, l)}^{H} \mathbf{h}_{r(k j)}\right|^{2}+\sum_{i=1}^{L} p_{k i}\left|\hat{\mathbf{w}}_{t(k, l)}^{H} \mathbf{c}_{r(k i)}\right|^{2}+r_{-k l}} \geq \gamma_{k l}
\end{aligned}
$$

where $\hat{\mathbf{w}}_{t(k, l)}=\frac{\mathbf{w}_{t(k, l)}^{*}}{\left\|\mathbf{w}_{t(k, l)}^{*}\right\|}$ is the normalized optimal transmit weight vector and $p_{k l}$ is the power used by radar- $k$ on the beam directed to target $l$. At this point, by redefining the acceptable strategy as $\mathcal{P}_{k}^{\prime}\left(\mathbf{p}_{-k}\right)=\left\{\mathbf{p}_{k} \in \mathbb{R}_{+}^{L} \mid \mathrm{SINR}_{k l} \geq\right.$ $\left.\gamma_{k l}, \forall l\right\}$ and the utility function as $u_{k}^{\prime}\left(\mathbf{p}_{k}\right)=\sum_{l=1}^{L} p_{k l}$, game $\mathcal{G}$ becomes a power allocation $\mathrm{SNG}$ :

$$
\mathcal{G}^{\prime}=\left\langle C,\left\{\mathcal{P}_{k}^{\prime}\left(\mathbf{p}_{-k}\right)\right\}_{k \in C},\left\{u_{k}^{\prime}\left(\mathbf{p}_{k}\right)\right\}_{k \in C}\right\rangle
$$

In order to prove the existence and the uniqueness of the NE of game G, we need to show that the best response strategy for every player is a standard function. We note that all the constraints must be active at the optimal power allocation. As a result, the inequality in the constraints of (6) can be replaced by equality and can be written as

$$
\mathbf{G}_{k} \mathbf{p}_{k}^{*}=\mathbf{r}_{-k}
$$

where $\mathbf{G}_{k} \in \mathbb{R}^{L \times L}$ and its elements are defined as $\left[\mathbf{G}_{k}\right]_{i i}=\left(\frac{\left|\hat{\mathbf{w}}_{t(k, i)}^{H} \mathbf{h}_{r(k i)}\right|^{2}}{\gamma_{k i}}-\left|\hat{\mathbf{w}}_{t(k, i)}^{H} c_{r(k i)}\right|^{2}\right)$ and $\left[\mathbf{G}_{k}\right]_{i j}=-\left|\hat{\mathbf{w}}_{t(k, i)}^{H} h_{r(k j)}\right|^{2}-\left|\hat{\mathbf{w}}_{t(k, i)}^{H} c_{r(k j)}\right|^{2}$, for $i \neq j$. The solution of (7) provides the optimal power allocation for (6). Following claim 2 in [12], the problem (6) is always feasible $\forall \mathbf{r}_{-k}>0$ elementwise. As a result, the matrix $\mathbf{G}_{k}$ must be invertible so we can straightforwardly obtain the best response strategy for the $k^{\text {th }}$ cluster as

$$
\mathbf{p}_{k}^{*}=\mathbf{G}_{k}^{-1} \mathbf{r}_{-k}
$$

The existence of the solution is guaranteed through the Arrow-Debreu theorem [33]. Since the NE exists, the uniqueness of this NE is proved by establishing that the best response function is standard [12]. We define the intercluster interference matrix from the $m^{\text {th }}$ radar to the $k^{\text {th }}$ radar as $\mathbf{G}_{m k} \in \mathbb{R}^{L \times L}$ and

$\left[\mathbf{G}_{m k}\right]_{i, j}=\left|\hat{\mathbf{w}}_{t(k, i)}^{H} \lambda_{r(k m j)}\right|^{2}+\left|\hat{\mathbf{w}}_{t(k, i)}^{H} \boldsymbol{\mu}_{r(k m)}\right|^{2}$. Hence, by replacing the interference vector $\mathbf{r}_{-k}$, we can restate the best response strategy as

$$
B R_{k}\left(\mathbf{p}_{-q}\right)=\mathbf{p}_{k}^{*}=\mathbf{G}_{k}^{-1}\left(\sum_{m \neq k}^{K} \mathbf{G}_{m k} \mathbf{p}_{m}^{*}+\mathbf{1}_{L} \sigma_{n}^{2}\right), \forall k
$$

where $\mathbf{1}_{L}$ denotes the all ones vector of size $L \times 1$.

LEMMA 1 The best response function (9) is a standard function.

PROOF The best response strategy (9) satisfies the following necessary properties for all $\mathbf{p} \geq 0$ : 
a) Positivity: $B R_{k}(\mathbf{p})>0$, as $\mathbf{G}_{k}^{-1}$ is a positive matrix straightforwardly from (8) and $\mathbf{G}_{m k}$ is a positive matrix from its definition.

b) Monotonicity: If $\mathbf{p} \geq \mathbf{p}^{\prime}$, then:

$$
B R_{k}(\mathbf{p})-B R_{k}\left(\mathbf{p}^{\prime}\right)=\mathbf{G}_{k}^{-1}\left(\sum_{m \neq k}^{K} \mathbf{G}_{m k}\left(\mathbf{p}_{m}-\mathbf{p}_{m}^{\prime}\right)\right) \geq 0 .
$$

c) Scalability: For all $a>1, a B R_{k}(\mathbf{p})>B R_{k}(\mathbf{a p})$. Indeed:

$$
a B R_{k}(\mathbf{p})-B R_{k}(a \mathbf{p})=(a-1) \mathbf{G}_{k}^{-1} \mathbf{1}_{L} \sigma_{n}^{2}>0 .
$$

By applying a pricing policy to each player we introduce some cooperation among them, which leads to a more Pareto efficient solution, as described in the next section.

\section{BEAMFORMER DESIGN AND POWER ALLOCATION GAME WITH PRICING}

A. Game Theoretical Formulation

Since each radar optimizes its beamformers and power allocation greedily, the equilibrium point is not necessarily the best solution from a social fairness point of view. This is explained because each player ignores the direct path interference it induces on other players. In order to obtain a more Pareto efficient solution and to increase the social welfare of the SNG, we introduce a pricing scheme applied to each radar's utility function. As a result, the players are encouraged to allocate their available resources more efficiently by minimizing the direct path interference induced to the other radars.

In order to achieve the aforementioned advantages, each radar/player needs to have information about the channel to the other radars in the system. Since we assume that the radars belong to the same organization, the knowledge of the channels between the radars is justified, as each radar knows the exact position of the others. Hence, each radar performs the following optimization:

$$
\begin{aligned}
& \min _{\mathbf{w}_{t(k)}} \sum_{l=1}^{L}\left\|\mathbf{w}_{t(k, l)}\right\|^{2}+\sum_{m \neq k}^{K} \sum_{i=1}^{L} \kappa_{k m i}\left\|\mathbf{w}_{t(k, i)} \boldsymbol{\mu}_{r(k m)}\right\|^{2} \\
& \text { s.t. } \frac{\left|\mathbf{w}_{t(k, l)}^{H} \mathbf{h}_{r(k l)}\right|^{2}}{\sum_{j \neq l}^{L}\left|\mathbf{w}_{t(k, l)}^{H} \mathbf{h}_{r(k j)}\right|^{2}+\sum_{i=1}^{L}\left|\mathbf{w}_{t(k, l)}^{H} \mathbf{c}_{r(k i)}\right|^{2}+r_{-k l}} \geq \gamma_{k l}, \forall l
\end{aligned}
$$

where $\kappa_{k m i}$ is the price charged to radar- $k$ for the interference it induces to radar $m$ when aiming at target $i$ and $\left\|\mathbf{w}_{t(k, i)} \boldsymbol{\mu}_{r(k m)}\right\|^{2}$ denotes the corresponding interference.

The aforementioned optimization encourages each player to adopt a more socially efficient power allocation strategy by steering its beampattern to the desired target, while keeping the sidelobes at the direction of the other players low and therefore causing less interference to other radars. As a result, the efficiency of the system as a whole is improved, yet the distributed nature of the game is preserved.

In order to reformulate the $\mathrm{SNG} \mathcal{G}$ to a more cooperative game with pricing consideration, we just need to redefine the utility function of radar- $k$ as $v_{k}\left(\mathbf{W}_{t(k)}\right)=\left\|\mathbf{W}_{t(k)}\right\|_{F}^{2}+\sum_{m \neq k}^{K} \sum_{i=1}^{L} \kappa_{k m i}\left\|\mathbf{w}_{t(k, i)} \boldsymbol{\mu}_{k m}\right\|^{2}$. The mathematical form of the pricing game is

$$
\mathcal{G}_{p r}=\left\langle C,\left\{\mathcal{P}_{k}\left(\mathbf{W}_{t(-k)}\right)\right\}_{k \in C},\left\{v_{k}\left(\mathbf{W}_{t(k)}\right)\right\}_{k \in C}\right\rangle .
$$

\section{B. Optimal Beamforming and the Best Response} Strategy

In this section, we design the optimal transmit and receive beamformers and the best response strategy for each of the players. Therefore, we exploit the fact that the optimization problem (10) can be reformulated as a convex optimization problem with second order cone (SOC) constraints [30]. This important property allows us to obtain the optimal solution via duality. The Lagrangian associated with the optimization problem (10) can be written as

$$
\begin{aligned}
\mathcal{L}\left(\mathbf{W}_{t(k)}, \lambda_{k}\right) & \\
= & \sum_{l=1}^{L}\left\|\mathbf{w}_{t(k, l)}\right\|^{2}+\sum_{m \neq k}^{K} \sum_{i=1}^{L} \kappa_{k m i}\left\|\mathbf{w}_{t(k, i)} \boldsymbol{\mu}_{r(k m)}\right\|^{2} \\
& +\sum_{l=1}^{L} \lambda_{k l}\left(\sum_{j \neq l}^{L}\left|\mathbf{w}_{t(k, l)}^{H} \mathbf{h}_{r(k j)}\right|^{2}+\sum_{i=1}^{L}\left|\mathbf{w}_{t(k, l)}^{H} \mathbf{c}_{r(k i)}\right|^{2}+r_{-k l}\right. \\
& \left.-\frac{1}{\gamma_{k l}}\left|\mathbf{w}_{t(k, l)}^{H} \mathbf{h}_{r(k l)}\right|^{2}\right)
\end{aligned}
$$

where $\lambda_{k}=\left[\lambda_{k 1}, \ldots, \lambda_{k L}\right]^{T}$ is the $L \times 1$ vector of the Lagrangian multipliers associated with the SINR inequality constraints of the problem in (10). The Lagrangian can be reorganized as

$$
\begin{aligned}
\mathcal{L}\left(\mathbf{W}_{t(k)}, \lambda_{k}\right)= & \sum_{l=1}^{L} \lambda_{k l} r_{-k l}+\sum_{l=1}^{L} \mathbf{w}_{t(k, l)}^{H}\left(\boldsymbol{\Omega}_{k}\left(\kappa_{k m l}\right)\right. \\
& -\frac{\lambda_{k l}}{\gamma_{k l}} \mathbf{h}_{r(k i)} \mathbf{h}_{r(k i)}^{H}+\sum_{j \neq l}^{L} \lambda_{k j} \mathbf{h}_{r(k j)} \mathbf{h}_{r(k j)}^{H} \\
& \left.+\sum_{i=1}^{L} \lambda_{k l} \mathbf{c}_{r(k i)} \mathbf{c}_{r(k i)}^{H}\right) \mathbf{w}_{t(k, l)}
\end{aligned}
$$

where $\boldsymbol{\Omega}_{k}\left(\kappa_{k m i}\right)=\sum_{m \neq k}^{K} \sum_{i=1}^{L} \kappa_{k m i} \boldsymbol{\mu}_{r(k m)} \boldsymbol{\mu}_{r(k m)}^{H}+\mathbf{I}$. At this point, we define the Lagrange dual function as the minimum value of the Lagrangian over $\mathbf{W}_{t(k)}$ :

$$
g_{k}\left(\boldsymbol{\lambda}_{k}\right)=\inf _{\mathbf{W}_{t(k)}} \mathcal{L}\left(\mathbf{W}_{t(k)}, \boldsymbol{\lambda}_{k}\right) .
$$

It is clear that if $\boldsymbol{\Omega}_{k}\left(\kappa_{k m l}\right)-\frac{\lambda_{k l}}{\gamma_{k l}} \mathbf{h}_{r(k i)} \mathbf{h}_{r(k i)}^{H}+$ $\sum_{j \neq l}^{L} \lambda_{k j} \mathbf{h}_{r(k j)} \mathbf{h}_{r(k j)}^{H}+\sum_{i=1}^{L} \lambda_{k l} \mathbf{c}_{r(k i)} \mathbf{c}_{r(k i)}^{H}$ is not positive semidefinite, the Lagrangian is unbounded below in $\mathbf{W}_{t(k)}$ and the dual function can take the value $-\infty$. Hence, the dual problem associated with (10) can be formulated as 


$$
\begin{gathered}
\max _{\lambda_{k 1}, \ldots, \lambda_{k L}} \sum_{l=1}^{L} \lambda_{k l} \boldsymbol{r}_{-k l} \\
\text { s.t. } \sum_{i=1}^{L} \lambda_{k l} \mathbf{h}_{r(k i)} \mathbf{h}_{r(k i)}^{H}+\sum_{i=1}^{L} \lambda_{k l} \mathbf{c}_{r(k i)} \mathbf{c}_{r(k i)}^{H}+\boldsymbol{\Omega}_{k}\left(\kappa_{k m l}\right) \\
\succeq\left(1+\frac{1}{\gamma_{k l}}\right) \lambda_{k l} \mathbf{h}_{r(k l)} \mathbf{h}_{r(k l)}^{H}, \forall l \\
\min _{\lambda_{k 1}, \ldots, \lambda_{k L}} \sum_{\mathbf{w}_{r(k, 1)}, \ldots, \mathbf{w}_{r(k, L)}}^{L} \lambda_{k l} r_{-k l} \\
\text { s.t. } \frac{\lambda_{k l}\left|\mathbf{w}_{r(k, l)}^{H} \mathbf{h}_{t(k l)}\right|^{2}}{\sum_{j \neq l}^{L} \lambda_{k j}\left|\mathbf{w}_{r(k, l)}^{H} \mathbf{h}_{t(k j)}\right|^{2}+\sum_{i=1}^{L}\left|\mathbf{w}_{r(k, l)}^{H} \mathbf{c}_{t(k i)}\right|^{2}+\mathbf{w}_{r(k, l)}^{H} \boldsymbol{\Omega}_{k}\left(\kappa_{k m i}\right) \mathbf{w}_{r(k, l)}} \geq \gamma_{k l}, \forall l \\
\lambda_{k l}^{(n+1)}=\frac{\gamma_{k l}}{1+\gamma_{k l}} \times \frac{1}{\mathbf{h}_{t(k l)}^{H}\left(\sum_{i=1}^{L} \lambda_{k l}^{n} \mathbf{h}_{t(k i)} \mathbf{h}_{t(k i)}^{H}+\sum_{i=1}^{L} \lambda_{k l}^{n} \mathbf{c}_{t(k i)} \mathbf{c}_{t(k i)}^{H}+\boldsymbol{\Omega}_{k}\left(\kappa_{k m l}\right)\right)^{-1} \mathbf{h}_{t(k l)}} .
\end{gathered}
$$

As mentioned in [34] and [12], where the authors investigate the downlink beamforming problem for communications application, the dual problem (11) is analogous to the receive beamforming optimization problem presented in (12).

Since the constraints are satisfied with equality at optimality, the optimal Lagrangian multipliers can be obtained by applying the fixed point iteration [34], as shown in (13). As proved in [34], the fixed point iteration described in (13) is shown to be a standard function and is guaranteed to converge to a unique solution, if the optimization problem (11) is feasible.

Subsequently, the optimal receive weight vector is the minimum mean-square error (MMSE) receiver, obtained as the following equation:

$$
\begin{aligned}
\mathbf{w}_{r(k, l)}= & \left(\sum_{i=1}^{L} \lambda_{k l} \mathbf{h}_{t(k i)} \mathbf{h}_{t(k i)}^{H}+\sum_{i=1}^{L} \lambda_{k l} \mathbf{c}_{t(k i)} \mathbf{c}_{t(k i)}^{H}\right. \\
& \left.+\boldsymbol{\Omega}_{k}\left(\kappa_{k m l}\right)\right)^{-1} \mathbf{h}_{t(k l)}
\end{aligned}
$$

Following [35], we can obtain the optimal transmit beamformer as a scaled version of the receive weight vector $\mathbf{w}_{t(k, l)}=\sqrt{\delta_{k, l}} \mathbf{w}_{r(k, l)}$, where $\delta_{k, l}$ is a scalar factor. The scaling factors $\delta_{k, l}$ can be found by exploiting the fact that the SINR constraints in (10) are met with equality at optimality. Hence by replacing $\mathbf{w}_{t(k, l)}=\sqrt{\delta_{k, l}} \mathbf{w}_{r(k, l)}$ into the SINR constraints, the scaling factors can be found from the following equation:

$$
\boldsymbol{\delta}_{\boldsymbol{k}}=\mathbf{F}^{-1} \mathbf{r}_{-k}
$$

where $\boldsymbol{\delta}_{\boldsymbol{k}}=\left[\delta_{k 1}, \delta_{k 2}, \ldots, \delta_{k L}\right]^{T}$ and $\mathbf{F} \in \mathbb{R}^{L \times L}$ and is defined as $[\mathbf{F}]_{i i}=\left(\frac{\left|\mathbf{w}_{r(k, i)}^{H} \mathbf{h}_{t(k i)}\right|^{2}}{\gamma_{k i}}-\left|\mathbf{w}_{r(k, i)}^{H} c_{t(k i)}\right|^{2}\right)$ and $[\mathbf{F}]_{i j}=-\left|\mathbf{w}_{r(k, i)}^{H} h_{t(k j)}\right|^{2}-\left|\mathbf{w}_{r(k, i)}^{H} c_{t(k j)}\right|^{2}$, for $i \neq j$.

Having decided the optimal transmit and receive beamformers, the solution of problem (10) is concluded. Similar to the game without pricing consideration, we can reformulate the initial optimization problem (10) as a power minimization problem. Following the same analysis as in Section III and by denoting the power vector of radar- $k$ as $\pi_{k} \in \mathbb{R}_{+}^{L}$, the best response strategy for the $k^{\text {th }}$ radar can be obtained from the following equation:

$$
\boldsymbol{\pi}_{k}^{*}=\boldsymbol{\Delta}_{k}^{-1} \mathbf{r}_{-k}
$$

where $\boldsymbol{\Delta}_{k} \in \mathbb{R}^{L \times L}$ and is defined as

$$
\begin{aligned}
& {\left[\boldsymbol{\Delta}_{k}\right]_{i i}=\left(\frac{\left|\mathbf{w}_{t(k, i)}^{H} \mathbf{h}_{r(k i)}\right|^{2}}{\gamma_{k i}}-\left|\mathbf{w}_{t(k, i)}^{H} c_{r(k i)}\right|^{2}\right) \text { and }} \\
& {\left[\boldsymbol{\Delta}_{k}\right]_{i j}=-\left|\mathbf{w}_{t(k, i)}^{H} h_{r(k j)}\right|^{2}-\left|\mathbf{w}_{t(k, i)}^{H} c_{r(k j)}\right|^{2} \text {, for } i \neq j .}
\end{aligned}
$$

Moreover, we denote the interradar interference matrix from the $m^{\text {th }}$ radar to the $k^{\text {th }}$ radar as $\boldsymbol{\Delta}_{m k} \in \mathbb{R}^{L \times L}$ and $\left[\boldsymbol{\Delta}_{m k}\right]_{i, j}=\left|\mathbf{w}_{t(k, i)}^{H} \lambda_{k m j}\right|^{2}+\left|\mathbf{w}_{t(k i)}^{H} \mu_{r(k m)}\right|^{2}$. Consequently, by replacing the interference vector

$\mathbf{r}_{-k}=\sum_{m \neq k}^{K} \boldsymbol{\Delta}_{m k} \mathbf{p}_{m}^{*}+\mathbf{1} \sigma_{n}^{2}$ we can redefine the best response strategy as

$$
B R_{k}\left(\boldsymbol{\pi}_{-k}\right)=\boldsymbol{\pi}_{k}^{*}=\boldsymbol{\Delta}_{k}^{-1}\left(\sum_{m \neq k}^{K} \boldsymbol{\Delta}_{m k} \boldsymbol{\pi}_{m}^{*}+\mathbf{1} \sigma_{n}^{2}\right), \forall k .
$$

LEMMA 2 The best response function (16) of the game with pricing consideration is a standard function.

Proof The proof is identical to that in lemma 1.

In the next section we present a hierarchical strategic game, known as the Stackelberg game. 


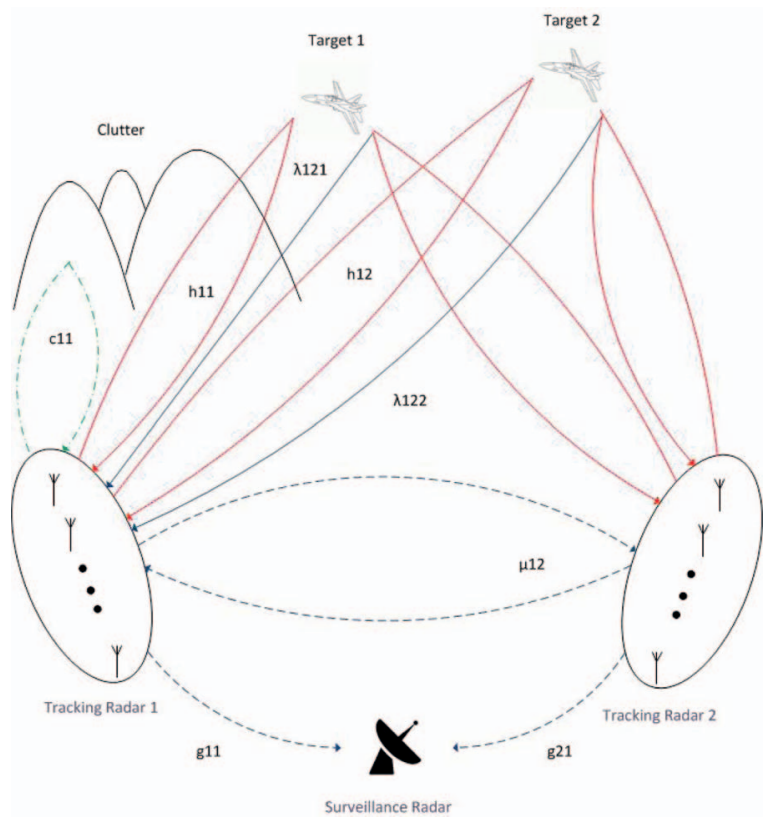

Fig. 2. Hybrid distributed MIMO radar network with surveillance radar, two tracking radars and two targets.

\section{STACKELBERG GAME SYSTEM MODEL}

In this section, we consider a hybrid MIMO network. More specifically, in addition to the multistatic tracking radar network mentioned in Section II, we incorporate a surveillance radar as part of the network, as seen in Fig. 2. We assume that all radars belong to the same organization and operate in the same field. As a result, the tracking radars may interfere with the surveillance radar and deteriorate its performance (increase the probability of false alarm). In order to guarantee the unimpeded operation of the system, the interference observed at the surveillance radar must not exceed a specific value, as shown below:

$$
\sum_{k=1}^{K} \sum_{l=1}^{L}\left|q_{r(s u r)}^{H} g_{k l}\right|^{2} \leq I_{\max }
$$

surveillance radar as observed from the $k^{\text {th }}$ tracking radar, and $I_{\max }$ is the maximum interference allowed. Since there is no transmit or receive beamformer at the surveillance radar, its receive filter $q_{r(s u r)}$ is a complex scalar.

In order to guarantee constraint (18), an interference cost can be imposed on every tracking radar in order to minimize their effect on the surveillance radar. Thus, a similar pricing mechanism to the previous section can be applied to every radar with the main objective to minimize the direct path interference to the surveillance radar. Owing to the fact that all radars belong to the same organization, we can safely assume that the information of the interradar channels is given. Similarly to the previous section, each tracking radar performs the following optimization:

$$
\begin{aligned}
& \min _{\mathbf{w}_{t(k)}} \sum_{l=1}^{L}\left\|\mathbf{w}_{t(k, l)}\right\|^{2}+\sum_{i=1}^{L} \kappa_{s u r}\left\|\mathbf{w}_{t(k, i)} \mathbf{g}_{k l}\right\|^{2} \\
& \text { s.t. } \frac{\left|\mathbf{w}_{t(k, l)}^{H} \mathbf{h}_{r(k l)}\right|^{2}}{\sum_{j \neq l}^{L}\left|\mathbf{w}_{t(k, l)}^{H} \mathbf{h}_{r(k j)}\right|^{2}+\sum_{i=1}^{L}\left|\mathbf{w}_{t(k, l)}^{H} \mathbf{c}_{r(k i)}\right|^{2}+r_{-k l}} \geq \gamma_{k l}, \forall l
\end{aligned}
$$

where $\kappa_{\text {sur }}$ is the pricing factor of interference, which is equally imposed by the surveillance radar to all tracking radars.

This interaction between the radars can be translated to a power allocation Stackelberg game, where the surveillance radar is the leader and the tracking radars are the followers. The strategy of the leader is the price of interference charged to the followers and the leader's utility function is its profit, which is defined as

$$
s_{\text {lead }}=\sum_{k=1}^{K} \sum_{l=1}^{L}\left|w_{r(s u r)}^{H} g_{k l}\right|^{2} \kappa_{\text {sur }}
$$

where $g_{k l}=\mathbf{w}_{t(k, l)}^{H} \mathbf{a}\left(\theta_{\operatorname{sur}(k)}\right)$ denotes the interfering signal in the direction of the surveillance radar when the $k^{\text {th }}$ tracking radar tags target $l, \theta_{\operatorname{sur}(k)}$ is the direction of the
Based on the price imposed by the leader, the followers decide their best response strategy as the result of the optimization in (19). 


\section{A. Followers' Game}

Since the followers know the price of interference announced by the leader, they decide their optimal beamformers and resource allocation by solving the optimization problem in (19). In order to formulate the followers' game, we observe that this game is similar to the game $\mathcal{G}_{p r}$, when we redefine the utility function of player $k$ as $s_{k}\left(\mathbf{W}_{t(k)}\right)=\left\|\mathbf{W}_{t(k)}\right\|_{F}^{2}+\sum_{i=1}^{L} \kappa_{s u r}\left\|\mathbf{w}_{t(k, i)} \mathbf{g}_{k l}\right\|^{2}$. Hence the mathematical representation of the followers' game is

$$
\mathcal{G}_{\text {fol }}=\left\langle C,\left\{\mathcal{P}_{k}\left(\mathbf{W}_{t(-k)}\right)\right\}_{k \in C},\left\{s_{k}\left(\mathbf{W}_{t(k)}\right)\right\}_{k \in C}\right\rangle .
$$

Following the same analysis as for game $\mathcal{G}_{p r}$, the optimal beamforming vectors can be derived by exploiting the duality properties of the convex optimization problem (18). Hence, respective to the receive weight vector optimization problem (12), we address the optimization problem in (21).

We denote $\bar{\lambda}_{k}=\left[\bar{\lambda}_{k 1}, \ldots, \bar{\lambda}_{k L}\right]^{T}$ as the $L \times 1$ vector of the Lagrangian multipliers associated with the inequality SINR constraints of problem (19), $\boldsymbol{\Omega}_{k}\left(\kappa_{s u r}\right)=\sum_{i=1}^{L} \kappa_{s u r} \mathbf{g}_{k l} \mathbf{g}_{k l}^{H}+\mathbf{I}$ and $\overline{\mathbf{w}}_{r(k, l)}$ as the $M \times 1$ receive weight vector for radar- $k$ regarding target- $l$ for the study of the Stackelberg game. Similar to (13), we obtain the optimal Lagrangian multipliers from (22) (the fixed point iteration in (22) is a standard function and admits a unique solution [34]) and the optimal receive beamformers through the MMSE receiver as

$$
\begin{aligned}
\overline{\mathbf{w}}_{r(k, l)}= & \left(\sum_{i=1}^{L} \bar{\lambda}_{k l} \mathbf{h}_{t(k i)} \mathbf{h}_{t(k i)}^{H}+\sum_{i=1}^{L} \bar{\lambda}_{k l} \mathbf{c}_{t(k i)} \mathbf{c}_{t(k i)}^{H}\right. \\
& \left.+\boldsymbol{\Omega}_{k}\left(\kappa_{\text {sur }}\right)\right)^{-1} \mathbf{h}_{t(k l)} .
\end{aligned}
$$

The optimal transmit beamformers are scaled versions of the optimal receive weight vectors:

$$
\overline{\mathbf{w}}_{t(k, l)}=\sqrt{\delta_{k, l}} \overline{\mathbf{w}}_{r(k, l)}
$$

Correspondingly to the method of $\mathcal{G}_{p r}$ and by indicating the power vector of radar- $k$ as $\boldsymbol{\rho}_{k} \in \mathbb{R}_{+}^{L}$, the best response strategy for the $k^{\text {th }}$ radar can be obtained from the following equation:

$$
\boldsymbol{\rho}_{k}^{*}=\boldsymbol{\Xi}_{k}^{-1} \mathbf{r}_{-k}
$$

where $\boldsymbol{\Xi}_{k} \in \mathbb{R}^{L \times L}$ and is defined as

$$
\begin{aligned}
& {\left[\boldsymbol{\Xi}_{k}\right]_{i i}=\left(\frac{\left|\overline{\mathbf{w}}_{t(k, i)}^{H} \mathbf{h}_{r(k i)}\right|^{2}}{\gamma_{k i}}-\left|\overline{\mathbf{w}}_{t(k i)}^{H} c_{r(k i)}\right|^{2}\right) \text { and }} \\
& {\left[\boldsymbol{\Xi}_{k}\right]_{i j}=-\left|\overline{\mathbf{w}}_{t(k i)}^{H} h_{r(k j)}\right|^{2}-\left|\overline{\mathbf{w}}_{t(k i)}^{H} c_{r(k j)}\right|^{2} \text {, for } i \neq j .}
\end{aligned}
$$

Furthermore, we denote the interradar interference matrix from the $m^{\text {th }}$ radar to the $k^{\text {th }}$ radar as $\boldsymbol{\Xi}_{m k} \in \mathbb{R}^{L \times L}$ and $\left[\boldsymbol{\Xi}_{m k}\right]_{i, j}=\left|\overline{\mathbf{w}}_{t(k i)}^{H} \lambda_{r(k m j)}\right|^{2}+\left|\overline{\mathbf{w}}_{t(k i)}^{H} \mu_{r(k m)}\right|^{2}$. Consequently, by replacing the interference vector $\mathbf{r}_{-k}=\sum_{m \neq k}^{K} \mathbf{\Xi}_{m k} \mathbf{p}_{m}^{*}+\mathbf{1} \sigma_{n}^{2}$ we can redefine the best response strategy as

$$
B R_{k}\left(\boldsymbol{\rho}_{-k}\right)=\boldsymbol{\rho}_{k}^{*}=\boldsymbol{\Xi}_{k}^{-1}\left(\sum_{m \neq k}^{K} \boldsymbol{\Xi}_{m k} \boldsymbol{\rho}_{m}^{*}+\mathbf{1} \sigma_{n}^{2}\right), \forall k .
$$

The study on the existence and the uniqueness of the solution is similar to the one in Section II.

\section{B. Leader's Game}

From the definition of the Stackelberg game, the leader knows the best response strategy of the followers.

Likewise in our model, the surveillance radar is aware of the existence of the tracking radars, as they belong to the same organization, and can determine the followers best response strategy. Hence, the leader's optimal strategy is extracted from the following optimization problem, where the leader's profit is maximized, while the interference is constrained under a maximum value to guarantee the efficient performance of the surveillance radar.

$$
\begin{aligned}
& \max _{\kappa_{\text {sur }}} \sum_{k=1}^{K} \sum_{l=1}^{L}\left|w_{r(\text { sur })}^{H} g_{k l}\right|^{2} \kappa_{\text {sur }} \\
& \text { s.t. } \quad \sum_{k=1}^{K} \sum_{l=1}^{L}\left|w_{r(\text { sur })}^{H} g_{k l}\right|^{2} \leq I_{\max }
\end{aligned}
$$

In order to determine the optimal price imposed by the leader to the tracking radars and solve the optimization problem (27), we adopt the learning algorithm for the leader as proposed in [16]. Initially, we determine the price $\kappa_{\text {sur }}^{*}$, where the constraint of the optimization problem (27) is met with equality:

$$
\sum_{k=1}^{K} \sum_{l=1}^{L}\left|w_{r(s u r)}^{H} g_{k l}\right|^{2}=I_{\max } .
$$

Hence, since the interference is a decreasing function of the price imposed by the leader, the constraint can be guaranteed when the price charged to the followers is not less than $\kappa_{\text {sur }}^{*}$, i.e., $\kappa_{\text {sur }} \geq \kappa_{\text {sur }}^{*}$. In algorithm 1 , we assume $\alpha$ is the learning rate of the algorithm $(\alpha>0)$ and $\kappa_{\text {sur }}^{t}$ is the price imposed by the leader at iteration $t$.

Algorithm 1: Learning algorithm for optimization problem (27)

1 Set an initial price $\kappa_{\text {sur }}^{1}=\kappa_{\text {sur }}^{*}$ determined at the equality of the constraint of optimization problem (27);

2 Determine an increment $\Delta \kappa_{\text {sur }}$ and set the second price value as: $\kappa_{\text {sur }}^{2} \kappa_{\text {sur }}^{*}+\Delta \kappa_{\text {sur }}$

3 Set $t=1$

4 while the convergence is not reached do:

5 Obtain the best response strategies for the tracking radars, by playing the followers game at price $\kappa_{\text {sur }}^{t}$

6 Calculate the profit of the leader $S_{\text {lead }}$ slead at price $\kappa_{\text {sur }}^{t}$

$7 \quad$ Determine the new price from the following learning equation:

$8 \quad \kappa_{\text {sur }}^{t+2}=\max \left(1+\alpha \frac{S_{\text {lead }}^{t+1}-S_{\text {lead }}^{t}}{\kappa_{\text {sur }}^{t+1}-\kappa_{\text {sur }}^{t}} \kappa_{\text {sur }}^{t+1}, \kappa_{\text {sur }}^{*}\right)$

9 Set $t=t+1$

10 end while 


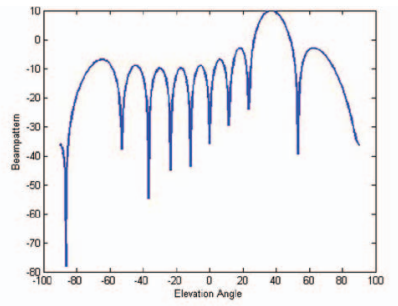

(a) Without pricing consideration.

Fig. 3. Comparison of transmit beampatterns for player 1 aiming at target $1(\mathrm{~dB})$

\section{SIMULATION RESULTS}

In this section, we present some simulation results to illustrate the performance of the beamformers and the convergence of the resource allocation methods for all three different games, which are the beamformer design and power allocation SNG, the beamformer design and power allocation game with pricing policy, and the Stackelberg game. Thus, we consider a bistatic network of two tracking MIMO radars, where each one consists of 10 transmit/receive antennas with half-wavelength spacing between adjacent antennas. The referential direction of the second radar as seen from the first radar is $\theta_{\operatorname{rad}(1,2)}=72^{\circ}$ and $\theta_{\operatorname{rad}(2,1)}=-75^{\circ}$ conversely. Moreover, we assume two targets placed at directions $\theta_{11}=37^{\circ}, \theta_{12}=22^{\circ}$ as observed from the first radar and $\theta_{21}=-38^{\circ}, \theta_{22}=-12^{\circ}$ using the second radar as reference. Furthermore, we assume strong clutter as a focal point with directions $\theta_{c l(1)}=52^{\circ}$ from radar 1 and $\theta_{c l(2)}=-54^{\circ}$ using the second radar as reference. The complex amplitudes of the targets and the clutter RCS are equal to $\beta_{1}=\beta_{2}=\beta_{c l}=1$. The background noise is considered as AWGN with variance 0.4 and the correlation factors between the waveforms for different targets $l \neq l^{\prime}$ are fixed to be equal to $0.1\left(\varrho_{k, l, l^{\prime}}=\varrho_{k, l, m, l^{\prime}}=0.1\right)$.

A. Comparison of the SNG and the Coordinated Game with Pricing Consideration

The first stage of the algorithm refers to the design of the optimal transmit and receive beamformers. In particular, for the SNG we obtain the aforementioned beamformers using convex SDP methods for the optimization problem (5), whereas for the coordinated game with pricing policy we exploit the duality properties of the optimization problem (10) and we find the transmit and receive weight vectors using the solution of the dual problem (14). It is obvious that in both games the beampatterns are concentrated on the desired target by maintaining very low sidelobe levels in other directions. Figs. 3-6 clearly depict the tendency towards social welfare of the game with pricing consideration, since the beampatterns of the first player enforce deep nulls at the direction of the other player, minimizing the interference leakage.

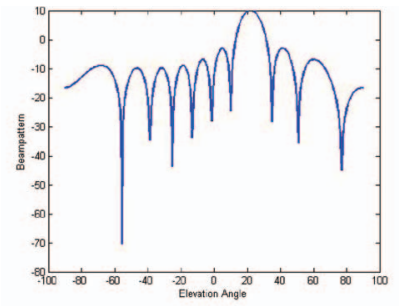

(a) Without pricing consideration.

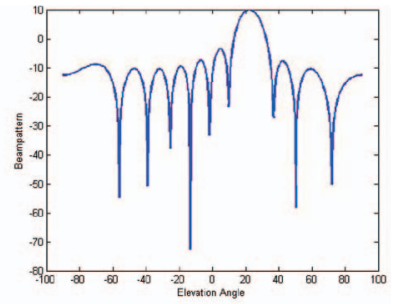

(b) With pricing consideration.
Fig. 4. Comparison of transmit beampatterns for player 1 aiming at target $2(\mathrm{~dB})$

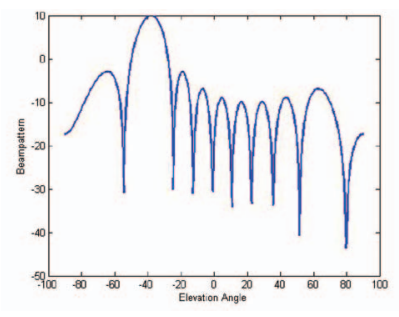

(a) Without pricing consideration.

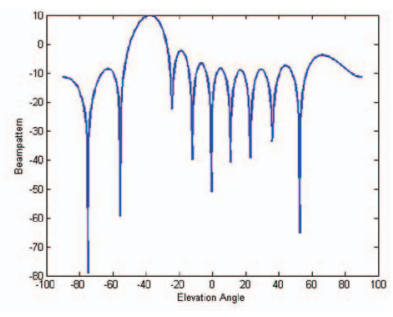

(b) With pricing consideration.
Fig. 5. Comparison of transmit beampatterns for player 2 aiming at target $1(\mathrm{~dB})$.

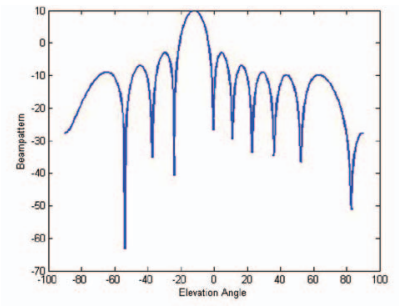

(a) Without pricing consideration.

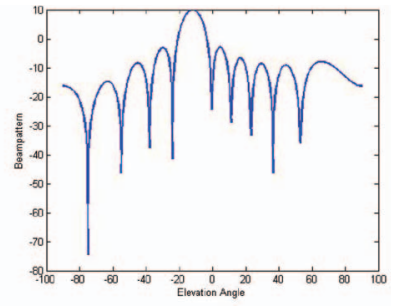

(b) With pricing consideration.
Fig. 6. Comparison of transmit beampatterns for player 2 aiming at target $2(\mathrm{~dB})$

The resource allocation optimization is considered at the second stage of the algorithms for both games compared. Before the initialization of the games, we decide the detection criterion for each player by setting the SINR targets at 7 for radar $1\left(\gamma_{11}=\gamma_{12}=7\right)$ and 6.5 for radar $2\left(\gamma_{21}=\gamma_{22}=6.5\right)$ for both games. We also set the maximum number of game iterations at $T=40$ to study the convergence of the algorithms. Figs. 8 and 9 depict the resource allocation update for each radar aiming each target. Power allocation using both methods clearly converges to a unique solution. Comparing Fig. 8 to Fig. 9 the advantages of the coordinated design with pricing are obvious, since the transmit power of each radar is lower compared with that of the SNG without pricing consideration. This result shows that due to the reduced interference among the radars using the coordinated design, as displayed in Fig. 7, each player needs less power to attain the SINR target, and hence the resource allocation for this game is more efficient. 


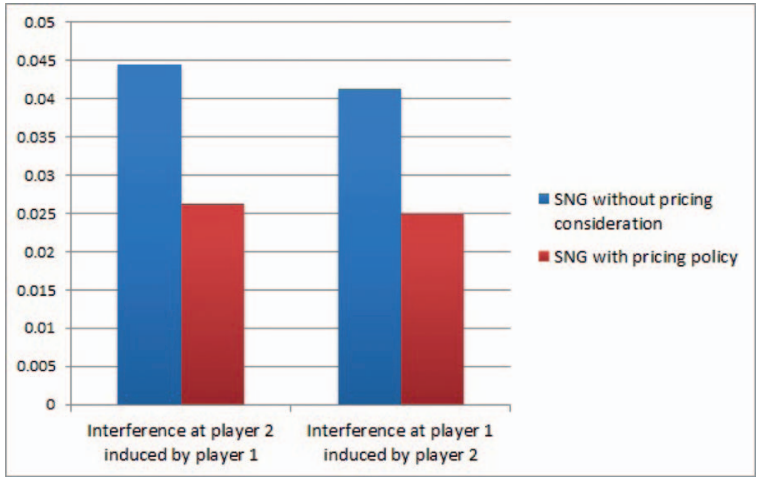

Fig. 7. Interference among MIMO tracking radars with and without pricing consideration.

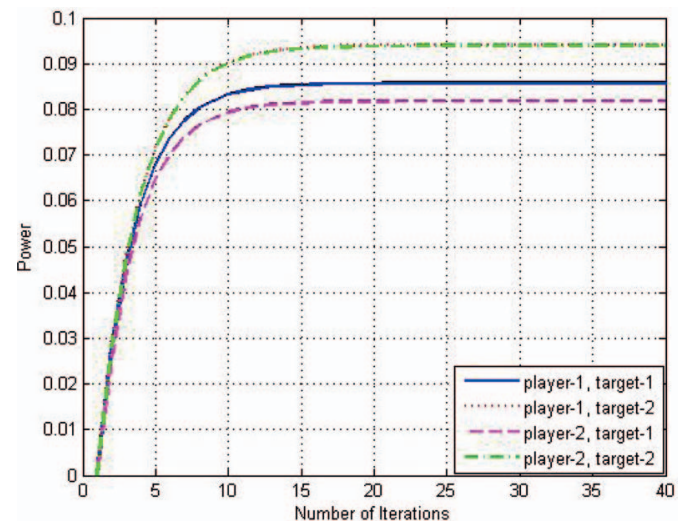

Fig. 8. Power allocation convergence for SNG without pricing consideration.

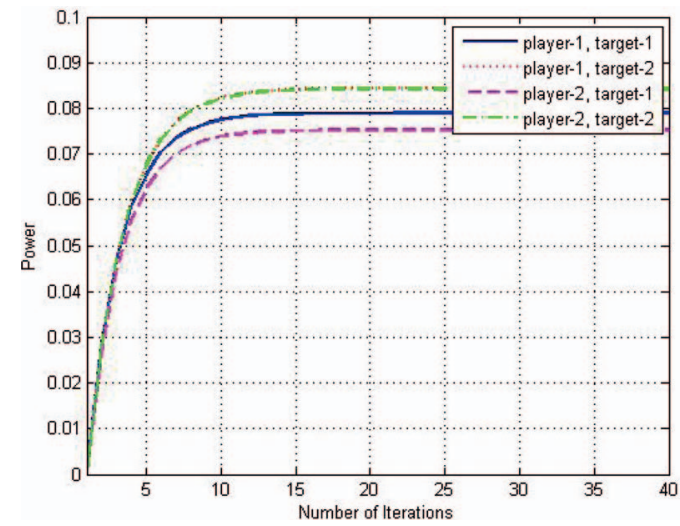

Fig. 9. Power allocation convergence for coordinated game with pricing policy.

\section{B. Stackelberg Game}

The surveillance radar is placed at direction $\theta_{\operatorname{sur}(1)}=$ $65^{\circ}$ as observed from the first tracking radar and $\theta_{\operatorname{sur}(2)}=$ $-67^{\circ}$ using the second radar as reference. Based on the price announced by the leader, the followers decide their optimal beamformers and power allocation by following game $\mathcal{G}_{\text {fol }}$. The transmit weight vectors and the power allocation of the followers, when the price set by the leader is $\kappa_{\text {sur }}=7.4$, are depicted in Figs. 10 and 11 and Fig. 12, respectively. It is clear that the beampatterns of

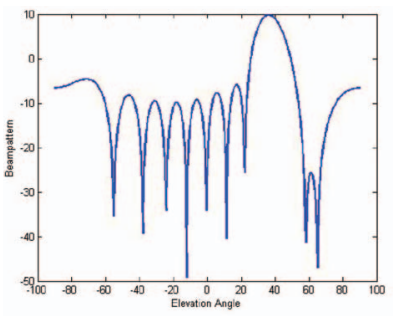

(a) Target $1(\mathrm{~dB})$.

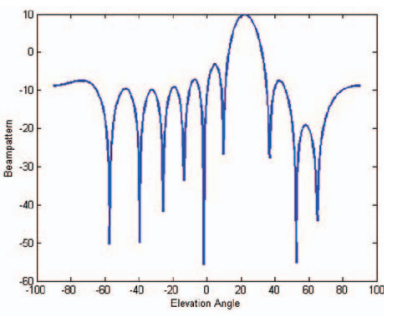

(b) Target $2(\mathrm{~dB})$.
Fig. 10. Transmit beampatterns for player 1 aiming at targets 1 and 2 respectively (Stackelberg game).

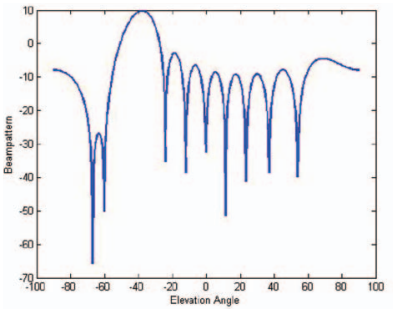

(a) Target $1(\mathrm{~dB})$.

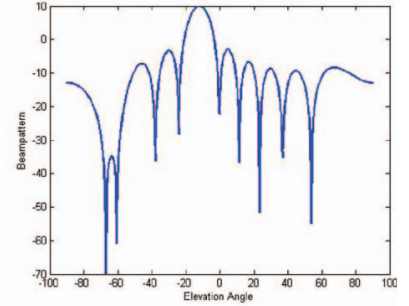

(b) Target $2(\mathrm{~dB})$.
Fig. 11. Transmit beampatterns for player 2 aiming at targets 1 and 2 respectively (Stackelberg game).

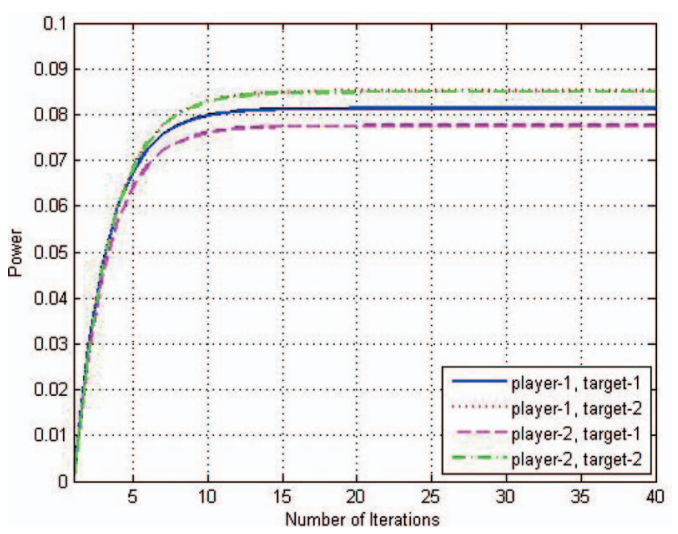

Fig. 12. Power allocation convergence for follower game when

$$
\kappa_{\text {sur }}=7.4 \text {. }
$$

both the followers are steered away from the direction of the leader and hence the interference leakage to the surveillance radar is minimized.

In order to find the optimal value of the price set by the leader, we solve the optimization problem in (27) incorporating the learning algorithm from Section V. We set the maximum interference allowed at the surveillance radar as $I_{\max }=0.0103$ and the learning rate as $\alpha=0.2$. For this interference threshold, the corresponding price is determined as $\kappa_{\text {sur }}=7.4$, which we consider as the initial price for the leader's game. The convergence of the price set by the leader is shown in Fig. 13. As expected, the algorithm rapidly converges to the starting price $\kappa_{\text {sur }}=$ 7.4, which is the minimum price so that the leader's interference constraint is secured. 


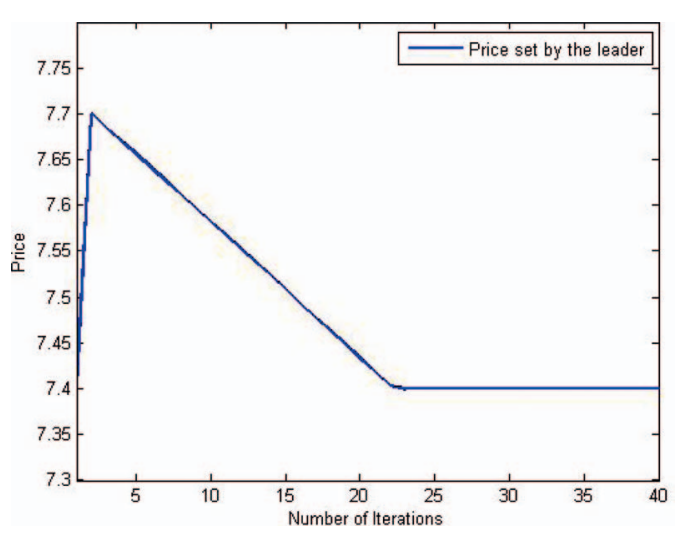

Fig. 13. Convergence of price imposed by leader.

\section{CONCLUSION}

We have investigated a game theoretic approach to tackle the problem of joint beamforming and power allocation in a distributed radar network. At first we studied an SNG, without any coordination among the radars/players. Thus each player greedily decides its optimal beamformers and power allocation. Furthermore, we incorporated a pricing mechanism to minimize the interradar interference and to improve the social welfare of the network. The simulation results confirm that this partially coordinated game provides a more Paretto-efficient NE. Additionally, we formulated a Stackelberg game by introducing a surveillance radar within the network and studied the convergence of both the followers' and the leader's games. Finally, the proofs for the existence and the uniqueness of both the partially coordinated and the noncooperative games have also been presented.

$$
\begin{aligned}
& \mathcal{L}\left(\mathbf{W}_{t(k)}, \lambda_{k}\right) \\
& =\sum_{l=1}^{L}\left\|\mathbf{w}_{t(k, l)}\right\|^{2}+\sum_{l=1}^{L} \lambda_{k l}\left(\sum_{j \neq l}^{L}\left|\mathbf{w}_{t(k, l)}^{H} \mathbf{h}_{r(k j)}\right|^{2}\right. \\
& \left.\quad+\sum_{i=1}^{L}\left|\mathbf{w}_{t(k, l)}^{H} \mathbf{c}_{r(k i)}\right|^{2}+r_{-k l}-\frac{1}{\gamma_{k l}}\left|\mathbf{w}_{t(k, l)}^{H} \mathbf{h}_{r(k l)}\right|^{2}\right)
\end{aligned}
$$

where $\lambda_{k}=\left[\lambda_{k 1}, \ldots, \lambda_{k L}\right]^{T}$ is the $L \times 1$ vector of the Lagrangian multipliers associated with the SINR inequality constraints of the problem in (10). The Lagrangian can be reformulated as

$$
\begin{aligned}
& \mathcal{L}\left(\mathbf{W}_{t(k)}, \lambda_{k}\right) \\
& =\sum_{l=1}^{L} \lambda_{k l} r_{-k l}+\sum_{l=1}^{L} \mathbf{w}_{t(k, l)}^{H}\left(\mathbf{I}-\frac{\lambda_{k l}}{\gamma_{k l}} \mathbf{h}_{r(k i)} \mathbf{h}_{r(k i)}^{H}\right. \\
& \left.\quad+\sum_{j \neq l}^{L} \lambda_{k j} \mathbf{h}_{r(k j)} \mathbf{h}_{r(k j)}^{H}+\sum_{i=1}^{L} \lambda_{k l} \mathbf{c}_{r(k i)} \mathbf{c}_{r(k i)}^{H}\right) \mathbf{w}_{t(k, l)} .
\end{aligned}
$$

Subsequently, we write the Lagrange dual function as

$$
g_{k}\left(\lambda_{k}\right)=\inf _{\mathbf{W}_{t(k)}} \mathcal{L}\left(\mathbf{W}_{t(k)}, \lambda_{k}\right) .
$$

It is clear that $\mathbf{I}-\frac{\lambda_{k l}}{\gamma_{k l}} \mathbf{h}_{r(k i)} \mathbf{h}_{r(k i)}^{H}+\sum_{j \neq l}^{L} \lambda_{k j} \mathbf{h}_{r(k j)} \mathbf{h}_{r(k j)}^{H}+$ $\sum_{i=1}^{L} \lambda_{k l} \mathbf{c}_{r(k i)} \mathbf{c}_{r(k i)}^{H}$ must be positive semidefinite, for the dual problem to be feasible. Hence, the dual problem associated with (5) can be designed as

$$
\begin{aligned}
& \max _{\lambda_{k 1}, \ldots, \lambda_{k L}} \sum_{l=1}^{L} \lambda_{k l} r_{-k l} \\
& \text { s.t. } \quad \sum_{i=1}^{L} \lambda_{k l} \mathbf{h}_{r(k i)} \mathbf{h}_{r(k i)}^{H}+\sum_{i=1}^{L} \lambda_{k l} \mathbf{c}_{r(k i)} \mathbf{c}_{r(k i)}^{H}+\mathbf{I} \\
& \succeq \frac{\lambda_{k l}}{\gamma_{k l}} \mathbf{h}_{r(k l)} \mathbf{h}_{r(k l)}^{H}, \forall l
\end{aligned}
$$

\section{APPENDIX}

PROOF OF ClAIM 1 In order to prove the optimal beampatterns independence of the interradar interference, we investigate the dual problem of the optimization problem (5). The Lagrangian associated with the aforementioned problem is given as
Following [34] and [12], the dual problem (30) can be solved through the receive beamforming optimization problem in (31).

Since the constraints are satisfied with equality at optimality, the optimal Lagrangian multipliers can be derived by applying the fixed point iteration method as in 
(32) [36]. It is also shown in [36] that the fixed point iteration function in (32) belongs to the framework of standard functions. Thus, the aforementioned iteration process is guaranteed to converge to a unique solution, if the respective optimization problem is feasible.

The optimal receive weight vector is the MMSE receiver, obtained from the following equation:

$$
\begin{aligned}
& \mathbf{w}_{r(k, l)} \\
& \quad=\left(\sum_{i=1}^{L} \lambda_{k l} \mathbf{h}_{t(k i)} \mathbf{h}_{t(k i)}^{H}+\sum_{i=1}^{L} \lambda_{k l} \mathbf{c}_{t(k i)} \mathbf{c}_{t(k i)}^{H}+\mathbf{I}\right)^{-1} \mathbf{h}_{t(k l)} .
\end{aligned}
$$

Following [35], the optimal transmit beamformer can be obtained as a scaled version of the receive weight vector $\mathbf{w}_{r(k, l)}$. Thus, it is clear that the optimal transmit and receive beampatterns are independent of the interradar plus noise vector $\mathbf{r}_{-k}$.

\section{REFERENCES}

[1] Li, J., and Stoica, P. MIMO Radar Signal Processing. New York: Wiley, 2009.

[2] Li, J., and Stoica, P. MIMO radar with colocated antennas. IEEE Signal Processing Magazine, 24, 5 (Sept. 2007), 106-114.

[3] Haimovich, A. M., Blum, R. S., and Cimini, L. J. MIMO radar with widely separated antennas. IEEE Signal Processing Magazine, 25, 1 (Jan. 2008), 116-129.

[4] Fuhrmann, D. R., Browning, J. P., and Rangaswamy, M. Signaling strategies for the hybrid MIMO phased-array radar. IEEE Journal of Selected Topics in Signal Processing, 4, 1 (Feb. 2010), 66-78.

[5] Tajer, A., Jajamovich, G. H., Wang, X., and Moustakides, G. V. Optimal joint target detection and parameter estimation by MIMO radar. IEEE Journal of Selected Topics in Signal Processing, 4, 1 (Feb. 2010), 127-145.

[6] Duly, A. J., Love, D. J., and Krogmeier, J. V. Time-division beamforming for MIMO radar waveform design.

IEEE Transactions on Aerospace and Electronic Systems, 4, 2 (Apr. 2013), 1210-1223.

[7] Saad, W., Han, Z., Debbah, M., Hjorungnes, A., and Basar, T. Coalitional game theory for communication networks. IEEE Signal Processing Magazine, 26, 5 (Sept. 2009), 77-97.

[8] Rashid-Farrokhi, F., Tassiulas, L., and Liu, K. J. R. Joint optimal power control and beamforming in wireless networks using antenna arrays. IEEE Transactions on Communications, 46, 10 (Oct. 1998), 1313-1324.

[9] Yu, W., Ginis, G., and Cioffi, J. M.

Distributed multiuser power control for digital subscriber lines.

IEEE Journal on Selected Areas in Communications, 20, 5 (June 2002), 1105-1115.

[10] Saraydar, C. U., Mandayam, N. B., and Goodman, D. J. Efficient power control via pricing in wireless data networks, IEEE Transactions on Communications, 50, 2 (Feb. 2002), 291-303.
[11] Saraydar, C. U., Mandayam, N. B., and Goodman, D. J. Pricing and power control in a multicell wireless data network, IEEE Journal on Selected Areas in Communications, 19, 10 (Oct. 2001), 1883-1892.

[12] Nguyen, D. H. N., and Le-Ngoc, T. Multiuser downlink beamforming in multicell wireless systems: A game theoretical approach. IEEE Transactions on Signal Processing, 59, 7 (July 2011), 3326-3338.

[13] Huang, J., Berry, R. A., and Honig, M. L.

Distributed interference compensation for wireless networks. IEEE Journal on Selected Areas in Communications, 24, 5 (May 2006), 1074-1084.

[14] Nguyen, D. N., and Krunz, M.

Power minimization in MIMO cognitive networks using beamforming games.

IEEE Journal on Selected Areas in Communications, 31, 5 (May 2013), 916-925.

[15] Wang, B., Han, Z., and Liu, K. J. R.

Distributed relay selection and power control for multiuser cooperative communication networks using Stackelberg game. IEEE Transactions on Mobile Computing, 8, 7 (May 2009), 975-990.

[16] Duong, N. D., Madhukumar, A. S., and Niyato, D.

Stackelberg Bayesian game for power allocation in two-tier networks.

IEEE Transactions on Vehicular Technology, 99 (Apr. 2015), $1-19$.

[17] Gogineni, S., and Nehorai, A.

Game theoretic design for polarimetric MIMO radar target detection.

Signal Processing, 92, 5 (2012), 1281-1289.

[18] Song, X., Willett, P., Zhou, S., and Luh, P.

The MIMO radar and jammer games.

IEEE Transactions on Signal Processing, 60, 2 (Feb. 2012), 687-699.

[19] Piezzo, M., Aubry, A., Buzzi, S., de Maio, A., and Farina, A. Non-cooperative code design in radar networks: A game-theoretic approach.

EURASIP Journal on Advances in Signal Processing, 2013, 1 (2013), 63.

[20] Panoui, A., Lambotharan, S., and Chambers, J. A. Game theoretic power allocation for a multistatic radar network in the presence of estimation error. In Sensor Signal Processing for Defence (SSPD), Sept. 2014.

[21] Bacci, G., Sanguinetti, L., Greco, M. S., and Luise, M. A game-theoretic approach for energy-efficient detection in radar sensor networks. In IEEE Sensor Array and Multichannel Signal Processing Workshop (SAM), June 2012.

[22] Sun, B., Chen, H., Wei, X., Wang, H., and Li, X.

Power allocation for range-only localisation in distributed multiple-input multiple-output radar networks - A cooperative game approach.

IET Radar, Sonar and Navigation, 8, 7 (Aug. 2014), 708-718.

[23] He, Q., Blum, R. S., Godrich, H., and Haimovich, A. M. Target velocity estimation and antenna placement for MIMO radar with widely separated antennas.

IEEE Journal of Selected Topics in Signal Processing, 4, 1 (Feb. 2010), 79-100.

[24] Gini, F.

Sub-optimum coherent radar detection in a mixture of $\mathrm{K}$-distributed and Gaussian clutter.

IEE Proceedings - Radar, Sonar and Navigation, 144, 1 (Feb. 1997), 39-48.

[25] Conte, E., Lops, M., and Ricci, G.

Asymptotically optimum radar detection in compound-Gaussian clutter. 
IEEE Transactions on Aerospace and Electronic Systems, 31, 2 (Apr. 1995), 617-625.

[26] Fuhrmann, D. R., and San Antonio, G.

Transmit beamforming for MIMO radar systems using signal cross-correlation.

IEEE Transactions on Aerospace and Electronic Systems, 44, 1 (Jan. 2008), 171-186.

[27] Hassanien, A., and Vorobyov, S. A.

Transmit energy focusing for DOA estimation in MIMO radar with colocated antennas.

IEEE Transactions on Signal Processing, 59, 6 (Mar. 2011), 2669-2682.

[28] Stoica, P., Li, J., and Xie, Y.

On probing signal design for MIMO radar.

IEEE Transactions on Signal Processing 55, 8 (Aug. 2007), 4151-4161.

[29] Deligiannis, A., Lambotharan, S., and Chambers, J. A.

Beamforming for fully-overlapped two-dimensional phased-MIMO radar.

IEEE Radar Conference (RadarCon), Arlington, VA, May 2015.

[30] Bengtsson, M., and Ottersen, B.

Optimal downlink beamforming using semidefinite optimization.

In Proceedings of 37th Annual Allerton Conference on

Communication, Control, and Computing, 1999.
[31] Luo, Z.-Q., Ma, W.-K., So, A. M.-C., Ye, Y., and Zhang, S. Semidefinite relaxation of quadratic optimization problems. IEEE Signal Processing Magazine, 27, 3 (May 2010), 20-34.

Yates, R. D.

A framework for uplink power control in cellular radio systems.

IEEE Journal on Selected Areas in Communications (Sept 1995).

[33] Arrow, K., and Debreu, G.

Existence of an equilibrium for a competitive economy. Econometrica, 3, 22 (1954), 256-290.

[34] Wiesel, A., Eldar, Y. C., and Shamai, S.

Linear precoding via conic optimization for fixed MIMO receivers.

IEEE Transactions on Signal Processing, 54, 1 (Jan. 2006), 161-176.

[35] Yu, W., and Lan, T.

Transmitter optimization for the multi-antenna downlink with per-antenna power constraints. IEEE Transactions on Signal Processing, 55, 6 (June 2007), 2646-2660.

[36] Dahrouj, H., and Yu, W.

Coordinated beamforming for the multicell multi-antenna wireless system.

IEEE Transactions on Wireless Communications, 9, 5 (May 2010), 1748-1759.
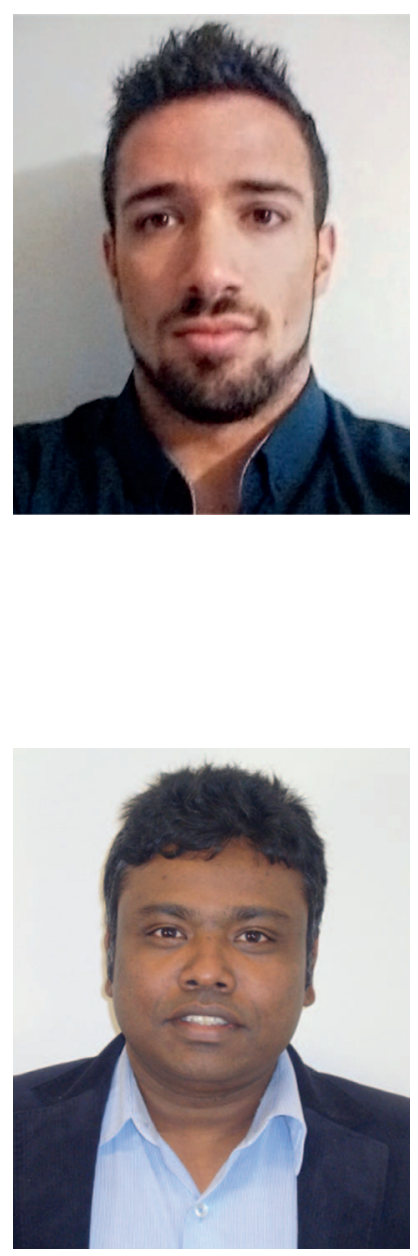

Anastasios Deligiannis (S'15) received his Diploma (Bachelor and Masters degrees equivalent) from the School of Electrical and Computer Engineering, University of Patras, Greece, in 2012. From 2013 he is a Ph.D. candidate within the Signal Processing and Networks Research Group, Wolfson School of Mechanical, Manufacturing and Electrical Engineering, Loughborough University, UK.

Since June 2016, he is a Research Associate in Signal Processing in Loughborough University. His research focuses on the design of signal processing algorithms, incorporating convex optimization and game theoretic methods, within the radar network framework.

Sangarapillai Lambotharan (SM'06) received his Ph.D. degree in signal processing from Imperial College London, UK, in 1997, and remained there until 1999 as a Postdoctoral Research Associate.

He was a Visiting Scientist in the Engineering and Theory Center of Cornell University, NY in 1996. From 1999 to 2002, he was with the Motorola Applied Research Group, UK, as a research engineer, working in many various projects, including physical-link layer modelling and performance characterization of GPRS, EGPRS, and UTRAN. From 2002 to 2007, he was with the King's College London, UK, and Cardiff University, UK, as a Lecturer and Senior Lecturer, respectively. He is currently Professor of Digital Communications and the Head of Signal Processing and Networks Research Group at Loughborough University, UK. His current research interests include wireless communications, cognitive radio networks, smart grids, radars, convex optimizations, and game theory.

Dr. Lambotharan has published over 175 conference and journal articles in the areas of his research interests. He serves as an Associate Editor for the EURASIP Journal on Wireless Communications and Networking. 


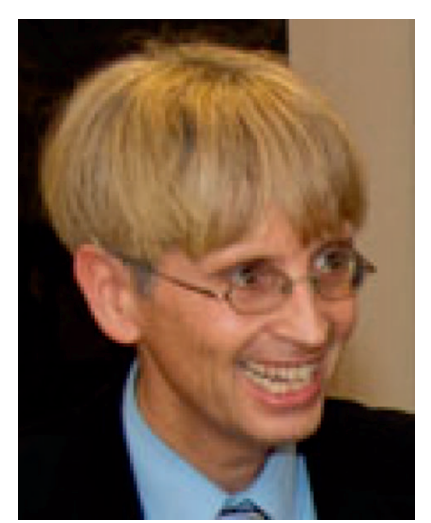

Jonathon Chambers (S'83-M'90-SM'98-F'11) received the Ph.D. and D.Sc. degrees in signal processing from the Imperial College of Science, Technology and Medicine (Imperial College London), London, UK, in 1990 and 2014, respectively.

From 1991 to 1994, he was a Research Scientist with Schlumberger Cambridge Research, Cambridge, UK. In 1994, he returned to Imperial College London as a Lecturer in signal processing and was promoted to Reader (Associate Professor) in 1998. From 2001 to 2004, he was the Director of the Center for Digital Signal Processing and a Professor of signal processing within the Division of Engineering, King's College London, and is now a Visiting Professor. From 2004 to 2007, he was a Cardiff Professorial Fellow with the School of Engineering, Cardiff University, Cardiff, UK. Between 2007-2014, he led the Advanced Signal Processing Group, within the School of Electronic, Electrical and Systems Engineering, Loughborough University, UK, and is now a Visiting Professor. In 2015, he joined the School of Electrical and Electronic Engineering, Newcastle Universtiy, where he is a Professor of signal and information processing and heads the ComS2IP group. He is also a Guest Professor at Harbin Engineering University.

Dr. Chambers has served as advisor to more than $70 \mathrm{Ph} . \mathrm{D}$. graduates and published more than 500 outputs. He is a Fellow of the Royal Academy of Engineering, the Institution of Electrical Engineers, and the Institute of Mathematics in its Applications in the UK. He has served as an Associate Editor for IEEE Transactions on Signal Processing for three terms over the periods 1997-1999, 2004-2007, and as a Senior Area Editor 2011-2015. 